\title{
MOSES HEALTH MONITORING SYSTEM FOR FIREFIGHTERS
}

\author{
Tatiana S. Goering-Zaburnenko ${ }^{1}$, Hilco Prins ${ }^{2}$ and Etto L. Salomons ${ }^{3}$ \\ ${ }^{1}$ Dr., Saxion University of Applied Science, Van Galenstraat 197500 KB Enschede, The Netherlands \\ ${ }^{2}$ Dr., Windescheim University of Applied Science, Campus 28017 CA Zwolle, The Netherlands \\ ${ }^{3}$ Ir., Saxion University of Applied Science, Van Galenstraat 197500 KB Enschede, The Netherlands
}

\begin{abstract}
When firefighting, the combination of exposition to high temperatures, high physical demands and wearing (heavy and insulated) personal protective equipment lead to increased risk of heat stress and exhaustion in firefighters. Heat stress can easily evolve into a life-threatening heat stroke. Once heat stress occurred, the chance of getting another heat stroke during deployment gets higher. Moreover, intermittent exposure to heat stress over several years, is a risk factor for heart diseases. Similarly, exhausted during a deployment, a firefighter needs more time to rehabilitate before he can safely be deployed again. Heat stress and exhaustion can lead to line-of-duty cardiovascular events. Therefore, preventing heat stress and exhaustion during deployment is beneficial for health, functioning and employability of firefighters. Since currently available measurement of the core temperature, such as thermometer pill or neck patch thermometer, are not reliable or practical for firefighters, an alternative approach may be used, namely, estimation of the core temperature based on non-invasive observation of the heart rate. Exhaustion is estimated using the training impulse model based on the heart rate reserve. Our achievement is a MoSeS health monitor system (as a smartphone application) that can real time analyze the health status of a firefighter and predict exhaustion and heat stress during deployment. The system is cheap (only a heart rate sensor and a smartphone application is needed), easy to use (intuitive "traffic light" signal), and objective (the health status is determined based on measurements of the heart rate). The only restriction is that the developed model is strongly dependent on personal maximum and minimum heart rate which need to be established beforehand.
\end{abstract}

\section{KEYWORDS}

Core Temperature, Heat Stress, Exhaustion, Firefighters

\section{INTRODUCTION}

Firefighters are regularly exposed to high temperatures which in combination with high physical demands and wearing (heavy and insulated) personal protective equipment (PPE) lead to higher risk of heat stress and exhaustion among firefighters (Barr, Gregson, \& Reilly, 2010). Heat stress occurs when body retains more heat than it loses. If heat stress is not treated properly, a life-threatening heat stroke may occur. A combination of fire heat, heat production of physical effort and insulation suit leads to a higher risk of heat stroke among firefighters. Heat stroke, besides being a direct danger to the physical health, has a negative impact on mental functioning, such as decision-making and responsiveness, which are crucial for firefighting. In particular, repeated exposure to heat stress causes health to be adversely affected (Hunter et al., 2017). Once heat stress is experienced chance of getting another heat stress during deployment gets higher (Druyan, Ketko, Yanovich, Epstein, \& Heled, 2013).

Exhaustion is another most common but also opaque hazard in fire fighters. Both heat stress and exhaustion may be a common causal factor in heart attacks and slips, trips and falls (Smith, Horn, Goldstein, \& Petruzzello, 2008). Heart failure is the cause of death in about half of the fatalities during firefighting in the USA (Kahn, Woods, \& Rae, 2015),(Sen, Palmieri, \& Greenhalgh, 2016),(Soteriades, Smith, Tsismenakis, Baur, \& Kales, 2011). Only the US has a national fire commissioner's office to compile statistics about line-of-duty-deaths. Overexertion / strain, and slips, trips and falls cause a large number of firefighter injuries ("National Fire Protection Association. Patterns of firefighter fireground injuries," 2016). While accidents and other calamities are rarely attributed to exhaustion, it is an influential factor in, for example, occurrences of accidents ("National Fire Protection Association. Patterns of firefighter fireground injuries," 2016). When a person is exhausted, coordination and alertness deteriorate, which can cause an accident. Models aimed to predict exhaustion primarily focus on prevention. It is important that a commander has a good insight in the health status of 
firemen primarily because they tend to neglect there body signals of tiredness and continue to do their work despite exhaustion or heat stress (Porter, 2000). If a model can objectively predict whether firefighters will be exhausted or overheated, actions can be taken by the commander to prevent this, for example, by instructing them to (temporarily) stop their activities and replacing them with 'fresh' firefighters.

The health monitoring system we developed is a part of the Mobile Sensing for Safety (MoSeS) system. The MoSeS system also determines the location of the firefighter within a building. It further establishes an ad hoc mobile network to communicate health status and location real-time with the commander. These parts of the system will not be discussed in this paper.

\section{MODEL DEVELOPMENT}

To be able to objectively predict the health status of a firefighter and make this visible to the commander in a natural manner we developed a model and implemented it into a "traffic light" smartphone application. The application shows the health status of a firefighter in colors red, orange, green or grey (ROGG status). Status "red" means that the firefighter is in a dangerous state of health, acute response from the commander is needed and the firefighter has to stop immediately with his work and leave the fire site. Status "orange" means that the firefighter has to be cautious with his activities as he threatens to be either exhausted or overheated. In this case the commander can give an order to temporarily stop his activities or slow down. Status "green" means that a firefighter has a good health condition and can continue his mission. Status "grey" means that the system is not able to evaluate the health status of the firefighter (e.g. no sensor signals were received or the received signals do not make sense, for example, when heart rate is shown to be zero but breathing rate is normal and an activity is detected).

\subsection{System Description}

An overview of the system is shown in Figure 1. Data from different sensors act as a system input. These heterogeneous data are transformed into a unified format ("InformationSource") and are stored in a central location on the smartphone of the firefighter ("Information System") together with the personal data of the firefighter ("Firefighters": age, gender, maximum heart rate, rest pulse, etc.). This information is then processed by two types of "Modules". "Aggregator Modules" aggregate data and store the results in the "Information System" (e.g. average heart rate per minute). "Analysis Modules" produce a health status (ROGG), using the data from the "Information System". These results are stored in the "Analysis System". The "Status Combiner" uses all results from the "Analysis Modules" to produce an overall health status (ROGG), which is then sent to the commander. The advantage of this architecture is the ability to add or remove diverse sensors and, if needed, change the "Module", "Information System" or "Analysis System".

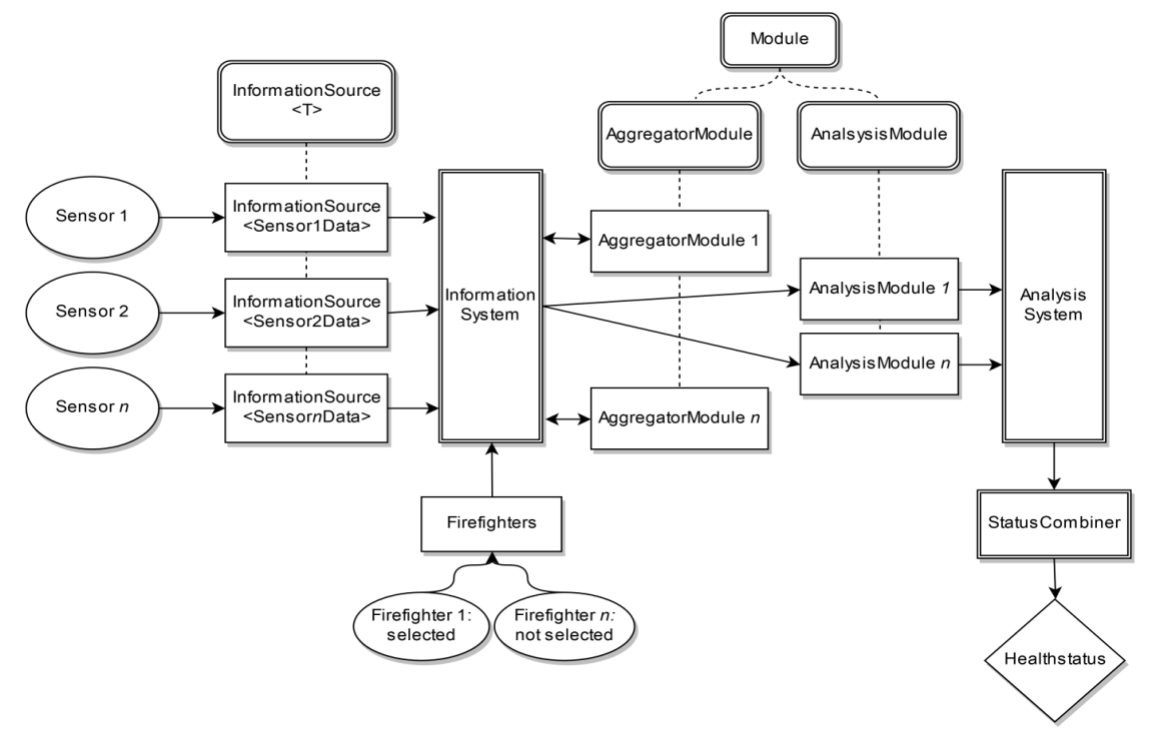

Figure 1. System Architecture Diagram 


\subsection{Heat Stress Monitoring}

Internal heat is stressful to the heart and can be a cause of acute heart problems, the most common cause of death by firefighters, especially if the heart is not completely healthy (Kahn et al., 2015), (Sen et al., 2016), (Soteriades et al., 2011). To be able to predict whether a firefighter is at risk of overheating is therefore of utmost importance. There are different ways to measure core temperature during physical activity ("A Pill that tracks your core body temperature using bluetooth technology and radio frequency," 2013),(Keene, Brearley, Bowen, \& Walker, 2015),(“The iDOT Body Temperature Alert Patch,” 2011). One of the possibilities is a thermometer pill ("Thermometer Pill' Helps Athletes, Firefighters and Other Beat the Heart," 2006). Although, theoretically speaking, the core temperature could be measured using a thermometer pill ("Thermometer Pill' Helps Athletes, Firefighters and Other Beat the Heart," 2006), it is not practical during fire fighting for at least two reasons. First, the thermometer pill is only effective two hours after being swallowed and performs best after at least six hours ("A Pill that tracks your core body temperature using bluetooth technology and radio frequency," 2013). Secondly, the thermometer pill is sensitive to drinking hot or cold drinks. Taking into account that a start of a firefighter's involvement is not known a priori and the fact that today's firefighters are engaged to regularly consume cold drinks to stimulate heat cooling and promote recovery, the thermometer pill cannot be used to reliably measure the core temperature of firefighters.

Other possibilities to measure core temperature could be an ear thermometer (Keene et al., 2015) or a patch behind the neck ("The iDOT Body Temperature Alert Patch," 2011). We did not choose the ear thermometer because its core temperature measurements are not yet reliable enough because of environmental influences (Keene et al., 2015). The patch behind the neck is also not yet practical for firefighters because it requires very careful placement and isolation of the sensor.

An alternative to measure body core temperature is to use an estimation model for body core temperature based on non-invasive observations of heart rate. Although these observations are very "noisy", it has been shown that body core temperature can be reliably estimated based on a discrete Kalman filter algorithm (Buller et al., 2013). The algorithm works as follows: initially, a body core temperature is defined (e.g. Tcore=37.1). Then a core temperature at the next moment is estimated using this input together with the current heart rate data (the heart rate is taken as a mean heart rate over a time period of one minute). This new core temperature estimation is used, together with the heart rate over the last minute, to predict body core temperature for the next minute (Buller et al., 2013). Research found that the algorithm's bias and variance to observed data were similar to that found from comparisons of esophageal and rectal measurements, both recognized but invasive and therefore impractical methods for measuring core temperature (Buller, Tharion, Duhamel, \& Yokota, 2015).

The advantage of this discrete Kalman Filter algorithm is its relative simplicity as it only requires one input (the heart rate) while other known models require more input parameters (Buller et al., 2013). Because of its simplicity, and the robustness and practical applicability of heart rate sensors (Buller et al., 2013), the Kalman filter algorithm was chosen to estimate body core temperature in our system.

\subsection{Prediction of Exhaustion}

The tasks associated with firefighting require aerobic fitness as well as anaerobic fitness (Barr et al., 2010). Various models have been developed for sport purposes to measure the impact of physical exercise on body. One of the measures that are used for this purpose is Training Impulse (TRIMP("TRIMP," 2015), ("Training Impulse," 2012)). Many different TRIMP models are known, from very simple, dependent only on the heart rate, to more complex models, dependent on the heart rate reserve (difference between personal maximum and minimum heart rate) or even more complicated, dependent on lactate levels ("TRIMP," 2015), ("Training Impulse," 2012). None of the models works optimal for all possible physical effort scenarios. The model that includes measurements of lactate levels could not be used in our system because it is impractical to measure lactate thresholds of all firefighters periodically by means of exercise tests. The next most sophisticated model is the exponential TRIMP model (TRIMPexp) based on the heart rate reserve ("TRIMP," 2015). It captures the fact that training at the higher heart rate is exponentially more intensive and thus leads to exponentially higher workload. Heart rate reserve is the difference between personal maximum and minimum heart rate (HRmax - HRrest). Normalised current heart rate reserve $(\mathrm{HRr})$, as a function of the actual heart rate (HRac), is given as follows: 


$$
H R r=\frac{H R a c-H R r e s t}{\text { HRmax }- \text { HRrest }}
$$

$H R r$ takes values between 0 and $1, H R r=0$ means that the current heart rate is equal to the heart rate at rest state $(H R a c=H R r e s t), H R r=1$ means that the current heart rate is at the maximum possible for this person, and e.g. $H R r=0.8$ means that the person is at $80 \%$ of his possible heart rate reserve.

The TRIMPexp model is described as follows ("TRIMP," 2015)

$$
T R I M P \exp =D * H R r * 0.64 * e^{C * H R r}
$$

where $H R r$ is being equal to the average heart rate reserve for time duration $D$ in minutes and $C$ is a gender-dependent constant based on a sample of males and females $(C=1.92$ for men and $C=1.67$ for women, ("TRIMP," 2015)). The TRIMPexp value can itself be normalized (TRIMPnorm) to a value between 0 and 10 based on the heart rate at rest $(H R r=0)$ and the heart rate at its maximum $(H R r=1)$ for TRIMPmin and TRIMPmax, respectively, given 1 minute of effort $(D=1)$.

$$
\begin{aligned}
& \text { TRIMPMin }=1 * 0 * 0.64 * e^{C * 0}=0 \\
& \text { TRIMPmax }=1 * 1 * 0.64 * e^{C * 1} \\
& (\approx 4.37 \text { for man and } \approx 3.40 \text { for woman }) \\
& \text { TRIMPnorm }=10 * \frac{\text { TRIMPexp }}{\text { TRIMPmax }}
\end{aligned}
$$

(TRIMPnorm gives a real time value between 0 and 10). Research has shown that an increase in heart rate in the higher zones (closer to the maximum heart rate) is heavier than an increase in heart rate in the lower zones (closer to the rest pulse) (Banister, Calvert, Savage, \& Bach, 1975), (Desgorces, Sénégas, Garcia, Decker, \& Noirez, 2007), (Morton, Fitz-Clarke, \& Banister, 1990). The TRIMPexp model used above captures that relationship (as described in ("TRIMP," 2015)) as the TRIMPexp value increases exponentially as the internal load of effort $(\mathrm{HRr})$ increases.

\subsection{Physiological Boundaries and Sensor Choice}

Physiological boundaries are personal boundaries of maximum and minimum heart rate (minimum heart rate is the rest pulse). Both personal HRmax and HRrest have to be established before the model can be used. The use of personalized $H R r$ is highly preferable, since then large individual differences in HRmax and HRrest are taken into account. It is important that these values are determined reliably and not based on a rule of thumb (such as 220 minus age for HRmax), this is due to the fact that many variation exists between individuals and thus deviations from the rule of thumb. It is also important to know that after an initial sharp increase in heart rates as a response to an alarm, heart rate only drops slightly while transported by the truck to the fire ${ }^{1}$ (Barr et al., 2010). Because in our system we use relatively high thresholds (which is not reached by the initial heart rate rise) this effect can be neglected.

As discussed above, models based on heart rate are good enough in estimating both heat stress and exhaustion. Using this knowledge and the restriction that sensors have to be usable under a firefighting suit, Zephyr Bioharness ("Zephyr BioHarness ${ }^{\mathrm{TM}}$ 3," n.d.) have been chosen to measure the heart rate of a firefighter.

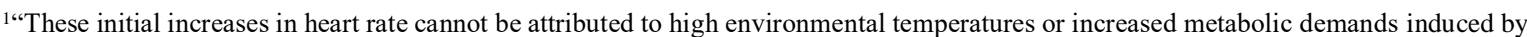
the addition of protective clothing, but more likely to be the combination of a sudden increase in physical activity and the high level of psychological stress. These findings emphasize the need for caution when using heart rate as a measurement during real-life fire-fighting activities as the relative contributions of the cardiovascular, nervous, and thermoregulatory system are difficult to determine." (Barr et al., 2010)
} 


\subsection{ROGG Model}

As our goal is to objectively determine the health status of a firefighter and to make it visible to the commander in a natural manner, a ROGG signal system was designed. As described above, prediction of heat stress as well as exhaustion is crucial for this purpose. The critical question is how we decide whether the health status is near to dangerous ("orange") or is already dangerous ("red"). To determine critical boundaries of different states ("red"/"orange"/"green") for internal heat and exhaustion, Ronald Heus, senior scientist at Institute for Safety, who is a specialist in physical performance of firefighters and personal protective equipment was consulted (Heus, n.d.). As a result, the following state diagram for internal heat was made

Figure 2 shows the critical value to make a transition from "green" to "orange" state as core temperature is $38^{\circ} \mathrm{C}$. The critical value to make a transition from "orange" to "red" state is the core temperature at $38.5^{\circ} \mathrm{C}$ (since we are talking about 'uncompensable' heatstress: a dangerous form due to the personal protective equipment.). As the core temperature drops below $38.5^{\circ} \mathrm{C}$ (respectively, $38^{\circ} \mathrm{C}$ ), the status goes back to "orange" (respectively, "green"). Because the core temperature does not change very quickly, we do not have to maintain a state long time and can make the transition immediately 2 .

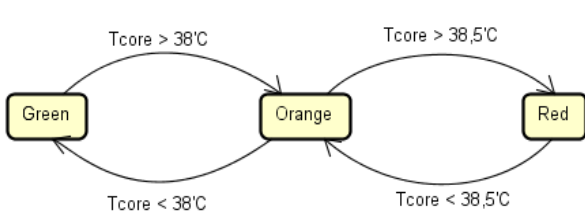

Figure 2. Internal heat state diagram

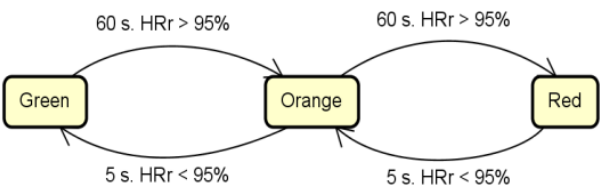

Figure 3. Very intensive short term effort

To predict exhaustion, we distinguish three possible risky health scenario's (Heus, n.d.):

1 Very intensive short term effort

2 Intensive medium term effort

3 Fairly intensive long term effort

Figures 3, 4 and 5 show when a transition from one state to another is made for short, medium and long term efforts. As it can be seen, when the heart rate reserve $(\boldsymbol{H} \boldsymbol{R} \boldsymbol{r})$ reaches $95 \%$ and stays there for at least one minute, the health status goes to "orange". If $\boldsymbol{H} \boldsymbol{R} \boldsymbol{r}$ is still at $95 \%$ for another minute then the health status goes to "red", when the $\boldsymbol{H} \boldsymbol{R} \boldsymbol{r}$ drops below $95 \%$ for at least five seconds the health status goes to "orange". If $\boldsymbol{H} \boldsymbol{R} \boldsymbol{r}$ remains below $95 \%$ for another five seconds, the health status goes to "green".

Figure 4 shows when a transition between different states is made in case of intensive medium term effort. If $\boldsymbol{H} \boldsymbol{R} \boldsymbol{r}$ stays above $90 \%$ for at least five minutes then the state becomes "orange". If $\boldsymbol{H} \boldsymbol{R} \boldsymbol{r}$ stays above $90 \%$ for another two minutes then the state goes to "red". The reverse (from "red" to "orange" and from "orange" to "green") transition occurs quicker as $\boldsymbol{H R r}$ drops below $90 \%$ for ten seconds. Figure 5 shows how decisions are made to make a transition between different states in case of fairly intensive long term effort. For this case, the earlier discussed TRIMP model (TRIMPnorm) is used because it better captures the changes in the higher heart rate reserve zones (due to exponential dependency on $H R r$; it also differ between men and women (see Eq.(2), (4)).

In case of fairly intensive long term effort the transition from "green" to "orange" state is made if the mean of TRIMPnorm for the last ten minutes exceeds TRIMPnorm for $80 \%$ of the heart rate reserve (TRIMPnorm as a function of $\boldsymbol{H R r}$ for $\boldsymbol{H R r}=\mathbf{0 . 8}$ ). If the mean of TRIMPnorm for another five minutes exceeds TRIMPnorm for $80 \%$ of the heart rate reserve, the state changes to "red". If the mean of TRIMPnorm for the duration of the last five minutes drops below TRIMPnorm for $80 \%$ of the heart rate reserve than the state changes to "orange", and if it does so for 'another' ten minutes than the state changes to "green".

${ }^{2} \mathrm{~A}$ validation study (Buller et al., 2013) concluded that the algorithm had a small bias $\left(0.02^{\circ} \mathrm{C}\right)$ and root mean square error $\left(0.21^{\circ} \mathrm{C}\right)$. Limits of agreement $\left(\mathrm{LoA} \pm 0.48^{\circ} \mathrm{C}\right)$ were similar to comparisons of $\mathrm{Tc}$ measured by oesophageal and rectal probes. These are reasonable estimates, but impact the reliability of our implementation. In order to prevent false negatives, we choose 'safe' limits for core temperature. 

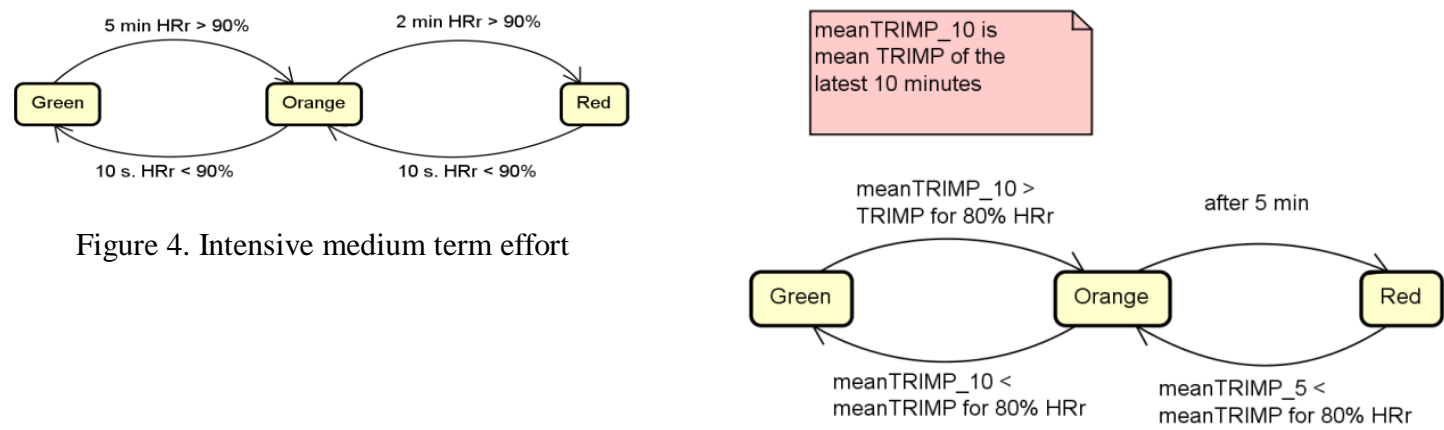

Figure 5. Fairly intensive long term effort

\subsection{Smartphone Application}

The final health status of a firefighter is a combination of internal heat and exhaustion. Our system is designed to be easy and transparent for the commander and shows the health status of a fireman in a glance. The Figure 6 shows how the smartphone application looks like for the commander. The commander sees on his tablet only one "red" (R)/ "orange" (O)/ "green" (G)/ "grey" (GR) signal (the box under "Health Status", orange in our case) for each firefighter under his command. The system monitors the state of all indicators. As soon as the ROGG signal on the commander's tablet turns 'orange' or 'red', the commander also receives information on his screen about which indicator is responsible for the warning.

The box under the health status of the smartphone application shows the reason why the health status is "orange". In the shown case, it is due to the short term very intensive effort. . In case the fireman is in risk of getting overheated or exhausted, the application immediately shows if it is due to temperature rise ("Temperature") or short term ("ShortTermveryIntensive"), medium term ("MediumTermIntensive") or long term ("LongTermTRIMP") effort. "BioHarnessBatteryLevel" shows the status of the battery, "ZephyrCommunication" shows if the Zephyr Bioharness ("Zephyr BioHarness TM 3," n.d.) is connected to the firefighter's smartphone and "StillRecieveingZephyrData" shows whether the Zephyr Bioharness is properly sending heart rate data to the smartphone. If the Zephyr Bioharnes is no longer properly connected to the smartphone ("ZephyrCommunication" is grey) or it is no longer sending heart rate data to the smartphone ("StillRecievingData" is grey) then the commander gets a "grey" signal, meaning that the system cannot properly determine the health status of a firefighter.

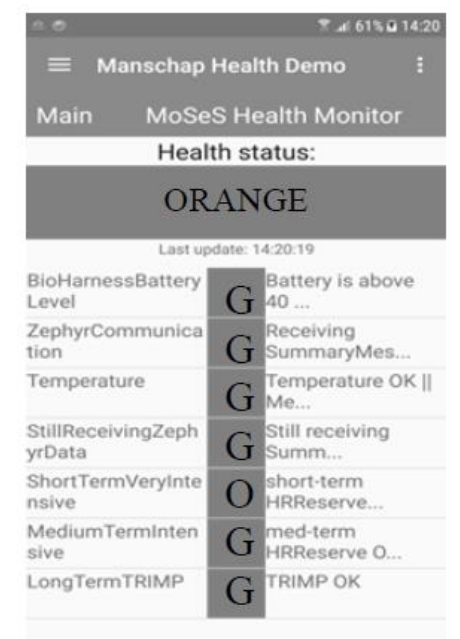

Figure 6. Snapshot smartphone application 


\section{CONCLUSION}

The following goals have been achieved:

1. We found out which key factors play role in the health risks of a firefighter (core temperature and exhaustion).

2. We found out the critical values $\left(38^{\circ} \mathrm{C} / 38.5^{\circ} \mathrm{C}\right)$ for the core temperature (when the core temperature reaches its first dangerous level $\left(38^{\circ} \mathrm{C}\right)$ the commander gets warned with "orange" signal, when the core temperature reaches its second dangerous level $\left(38.5^{\circ} \mathrm{C}\right)$ the commander gets warned with "red" signal).

3. We investigated existing ways of measuring or estimating core temperature and found out that estimating the core temperature using Kalman filter algorithm based on the heart rate gives reliable results for predicting core temperature (Buller et al., 2013).

4. We distinguished three different sorts of intensity efforts that contribute to exhaustion (very intensive short term effort, intensive medium term effort and fairly intensive long term effort) and critical thresholds for warnings towards the commander (first, an "orange" signal to indicate that a firefighter is going to get exhausted, and then a "red" signal when exhaustion gets really dangerous).

5. We have developed a modular system to combine different health outcomes to predict the health status of a firefighter. The system is not dependent on the chosen sensor (Zephyr Bioharness, ("Zephyr BioHarness ${ }^{\mathrm{TM}} 3$," n.d.)) and can be easily changed if more reliable sensors are available on the market. It is also possible to add other sensors to the system if research shows that more reliable outcomes can be reached with extra or other sensor information,

Last but not least, we developed the smartphone application (Laarhuis, 2018) which gives objective information about the health status of a firefighter in a transparent and easy way (ROGG ("red"/ "orange"/ "green"/ "grey") signal).

\subsection{Restrictions}

- In general to be able to draw proper conclusions a system needs to be validated. In our case that means that enough firefighters have to be put in a situation when they achieve their maximum heart rate or are exposed to dangerous core temperature rise. Because heat stress is extremely dangerous and, moreover, increases the chance of getting another heat stress once exposed to one, it is dangerous as well as not ethical to expose firefighters to these extreme conditions in laboratory settings. That makes validation of the system not easy ${ }^{3}$.

- $\quad$ Our model for predicting exhaustion is strongly dependent on personalised maximum heart rate and rest pulse. If personal data are not available our system cannot be used properly because of high variability of heart rate boundaries (maximum and minimum heart rates).

The developed MoSeS health monitor system can analyze the health status of a firefighter and predict exhaustion and heat stress real-time during deployment ("orange" signal). Above that, the system is cheap (no extra investments are needed besides the smartphone application and a heart rate monitor ("Zephyr BioHarness TM 3," n.d.)), easy to use (intuitive "traffic light" signal), and objective (the health status is determined based on the heart rate). The health monitoring system is a part of a larger safety awareness and communication system MoSeS (Laarhuis, 2018) which not only determines health status of a firefighter but also establishes an ad hoc mobile network to determine position of a firefighter within a building. As a proof of concept the technical working of the complete system was demonstrated in a simulated emergency operation.

\section{ACKNOWLEDGEMENTS}

We wish to acknowledge the Fire Department Twente ("Brandweer Twente," n.d.) for their collaboration and providing necessary data for our research. We are also thankful for the advice given by Ronald Heus, a specialist in physical performance and personal protective equipment (Heus, n.d.). He has been a great help in determining critical values for the core temperature as well as for determining three possible risky health scenario's (see Chapter 2.5). Last but not least, we wish to thank the sponsor of the project Tech For Future ("Tech For Future," n.d.). Without the sponsoring the whole project would be impossible.

${ }^{3}$ In (Buller et al., 2013) the algorithm was validated in tests with first responders wearing personal protective equipment. We used HR data from periodical preventive medical tests and theoretical scenario's to study the behavior of our system and simulated an emergency operation in which the technical working of the system was demonstrated. 


\section{REFERENCES}

A Pill that tracks your core body temperature using bluetooth technology and radio frequency. (2013). Retrieved from http://www.mysuncoast.com/news/health_smart/a-pill-that-tracks-your-core-body-temperature-usingbluetooth/article_dec5a8ee-e35b-11e2-a472-0019bb30f31a.html

Banister, E. W., Calvert, I. W., Savage, M. V., \& Bach, I. M. (1975). A system model of training for athletic performance. Australian Journal of Sports Medicine, 7, 170-176.

Barr, D., Gregson, W., \& Reilly, T. (2010). The thermal ergonomics of firefighting reviewed. Applied Ergonomics, 41(1), 161-172. https://doi.org/10.1016/j.apergo.2009.07.001

Brandweer Twente. (n.d.). Retrieved from https://www.brandweer.nl/twente

Buller, M. J., Tharion, W. J., Cheuvront, S. N., Montain, S. J., Kenefick, R. W., Castellani, J., ... Hoyt, R. W. (2013). Estimation of human core temperature from sequential heart rate observations. Physiological Measurement, 34(7), 781-798. https://doi.org/10.1088/0967-3334/34/7/781

Buller, M. J., Tharion, W. J., Duhamel, C. M., \& Yokota, M. (2015). Real-time core body temperature estimation from heart rate for first responders wearing different levels of personal protective equipment. Ergonomics, 58(11), 1830-1841. https://doi.org/10.1080/00140139.2015.1036792

Desgorces, F.-D., Sénégas, X., Garcia, J., Decker, L., \& Noirez, P. (2007). Methods to quantify intermittent exercises. Applied Physiology, Nutrition, and Metabolism, 32(4), 762-769. https://doi.org/10.1139/H07-037

Druyan, A., Ketko, I., Yanovich, R., Epstein, Y., \& Heled, Y. (2013). Refining the distinction between heat tolerant and intolerant individuals during a Heat tolerance test. Journal of Thermal Biology, 38(8), 539-542. https://doi.org/10.1016/j.jtherbio.2013.09.005

Heus, R. (n.d.). Senior Scientist at Institute for Safety IFV. Retrieved from https://www.linkedin.com/in/ronaldheus/

Hunter, A. L., Shah, A. S. V., Langrish, J. P., Raftis, J. B., Lucking, A. J., Brittan, M., ... Mills, N. L. (2017). Fire simulation and cardiovascular health in firefighters. Circulation, 135(14), 1284-1295. https://doi.org/10.1161/CIRCULATIONAHA.116.025711

Kahn, S. A., Woods, J., \& Rae, L. (2015). Line of duty firefighter fatalities: An evolving trend over time. Journal of Burn Care and Research, 36(1), 218-224. https://doi.org/10.1097/BCR.0000000000000104

Keene, T., Brearley, M., Bowen, B., \& Walker, A. (2015). Accuracy of tympanic temperature measurement in firefighters completing a simulated structural firefighting task. Prehospital and Disaster Medicine, 30(5), 461-465. https://doi.org/10.1017/S1049023X15005038

Laarhuis, J. (2018). MoSeS ondersteunt brandweer. NiDV, 23-25. Retrieved from https://www.nidv.eu/wpcontent/uploads/2018/03/NIDV_magazine_2018-1.pdf

Morton, R. H., Fitz-Clarke, J. R., \& Banister, E. W. (1990). Modeling human performance in running. Journal of Applied Physiology, 69(3), 1171-1177. https://doi.org/10.1152/jappl.1990.69.3.1171

National Fire Protection Association. Patterns of firefighter fireground injuries. (2016). Retrieved from http://www.nfpa.org/news-and-research/fire-statistics-and-reports/fire-statistics/the-fire-service/fatalities-andinjuries/patterns-of-firefighter-fireground-injuries

Porter, A. M. W. (2000). The death of a British officer-cadet from heat illness. Lancet, 355(9203), $569-571$. https://doi.org/10.1016/S0140-6736(99)06407-7

Sen, S., Palmieri, T., \& Greenhalgh, D. (2016). Cardiac fatalities in firefighters: An analysis of the U.S. fire administration database. Journal of Burn Care and Research, 37(3), 191-195. https://doi.org/10.1097/BCR.0000000000000225

Smith, D. L., Horn, G., Goldstein, E., \& Petruzzello, S. J. (2008). Firefighter Fatalities and Injuries: The Role of Heat Stress and PPE. Retrieved from https://www.fsi.illinois.edu/documents/research/FFLSRC_FinalReport.pdf

Soteriades, E. S., Smith, D. L., Tsismenakis, A. J., Baur, D. M., \& Kales, S. N. (2011). Cardiovascular disease in US firefighters: A systematic review. Cardiology in Review, 19(4), 202-215. https://doi.org/10.1097/CRD.0b013e318215c105

Tech For Future. (n.d.). Retrieved from http://techforfuture.nl/

The iDOT Body Temperature Alert Patch. (2011). Retrieved from http://www.johnsonlambe.net/features-specials/idotpatch.htm

Thermometer Pill' Helps Athletes, Firefighters and Other Beat the Heart. (2006). Retrieved from https://www.nasa.gov/topics/nasalife/thermometer_pill.html

Training Impulse. (2012). Retrieved from http://www.trainingimpulse.com/

TRIMP. (2015). Retrieved from http://fellrnr.com/wiki/TRIMP

Zephyr BioHarness ${ }^{\mathrm{TM}}$ 3. (n.d.). Retrieved from https://www.zephyranywhere.com/ 